(198)

\title{
Prevalence of Antibiotic Resistant Bacteria in Fecal Matter of Wild Elephants (Elephas maximus)
}

\author{
Jayasekara J.A.A.W.L. ${ }^{*}$, Abeysinghe K.G.D.De.A. ${ }^{1}$, Dissanayake D.M.J.C. ${ }^{2}$, \\ Vandercone R.P.G. ${ }^{1}$, Bamunuarachchige T.C. ${ }^{1}$ \\ ${ }^{1}$ Department of Biological Sciences, Rajarata University, Sri Lanka \\ ${ }^{2}$ Teaching Hospital, Anuradhapura, Sri Lanka. \\ *wimanilj@gmail.com
}

\begin{abstract}
Excessive usage of antibiotics in medicinal and non-medicinal fields such as agriculture, animal husbandry and aquaculture is the fundamental cause for developing antibiotic resistance in the wild. When wild animals acquire antibiotic resistant bacteria, they can act as reservoirs which provides a biological mechanism in disseminating the antibiotic resistant bacteria into a vast range of environmental niches. The objective of the study was to determine the prevalence of antibiotic resistant bacteria in wild elephants (Elephas maximus) as they range in a variety of territories while showing increased proximity to human activities. Fecal samples of elephants were collected from Kaudulla National Park, Sri Lanka in February, 2017. Cary Blair Transport Medium was used for the transportation, preservation and examination of fecal samples for enteric pathogens. The bacterial population was isolated and identified by Ribotyping and biochemical tests. Antibiotic Susceptibility Test (ABST) was done to identify antibiotic resistant patterns in bacteria. Quality control strain, Escherichia coli ATCC 25922 (provided by MRI, Colombo, Sri Lanka) was used to validate the routine susceptibility testing methods. ABST was performed by using Kirby-Bauer disk diffusion method for isolated bacterial strains; Eschrichia hermannii, E. coli, Enterobacter sp., E. asburiae, E. sacchari and Bacillus cereus. Antibiotics used were Ampicillin (AMP), 10 $\mu \mathrm{g}$; Amoxicillin-clavulanic acid (AMC), $30 \mu \mathrm{g}$; Ceftriaxone (CRO), $30 \mu \mathrm{g}$; Cefuroxime (CXM), $30 \mu \mathrm{g}$; Imipenem (IMP), $10 \mu \mathrm{g}$; Meropenem (MEM), $10 \mu \mathrm{g}$; Gentamicin (CN), 10 $\mu \mathrm{g}$; Amikacin (AK), $30 \mu \mathrm{g}$; Tetracycline (TE), $30 \mu \mathrm{g}$; Ciprofloxacin (CIP), $5 \mu \mathrm{g}$; Norfloxacin (NOR), $10 \mu \mathrm{g}$ and Nitrofurantoin (F), $300 \mu \mathrm{g}$. The results show that all bacterial isolates of Family Enterobacteriaceae are resistant or intermediately resistant at least for one antibiotic used in the study. The test organisms were susceptible for majority of the antibiotics except for Ampicillin (AMP), Amoxicillin-clavulanate (AMC), Tetracycline (TE), Nitrofurantoin (F) and Cefuroxime (CXM). Organisms that show resistance for more than one antibiotic are considered to show Multi Drug Resistance. Accordingly E. hermannii and all the Enterobacter spp. are multidrug resistant strains. Nitrofurantoin resistance and ampicillin resistance were the predominant resistance shown by bacteria. Exhibited antibiotic resistance shows the prevalence of antibiotic resistance in gut microflora of wild elephants.
\end{abstract}

Keywords: Antibiotic resistant bacteria, Wild elephants, Enterobacteriaceae, Escherichia, Enterobacter

Proceedings of the $22^{\text {nd }}$ International Forestry and Environment Symposium 2017 of the Department of Forestry and Environmental Science, University of Sri Jayewardenepura, Sri Lanka 